\title{
Editorial
}

\section{Oncoplastic surgery}

\author{
Nicolae Ghețu, MD, PhD, \\ Guest Editor
}

Assist. Prof., Department of Plastic Surgery, "Grigore T. Popa" University of Medicine and Pharmacy, lași; Regional Institute of Oncology, lași, Romania

A few decades ago, oncologic surgeons coined the term "oncoplastic" to operations designed to safely remove breast cancer following the oncologic principles, at the same time avoiding the mutilating radical mastectomy. Oncologic principle means removing the disease with adequate margins so as to prevent or decrease to a virtual minimum the persistence of disease or the recurrences. Concomitantly, the remaining breast tissues would be re-arranged, reshaped or supplemented with autologous or alloplastic materials in order to maintain breast shape, and as much as possible, function and beauty, even throughout the adjuvant therapy. Ever since, oncoplastic breast conserving surgery made the proof of concept, and not only surpassed the limitations but pushed the advantages forward. Thus, several recent meta-analysis reported oncoplastic breast surgery successful in achieving a normalappearing breast and similar oncologic results with mastectomy or other breast conserving techniques.

However, the present issue of Archive of Clinical Cases is not about breast oncoplastic surgery. It is but a mere invitation to observe how oncoplastic principles apply to other oncologic scenarios.

Wide excision for cancer leaves patients with defects that add to the psychological trauma of an oncologic disease. With the advances in cancer therapy leading to increased healing rates, patients' attention turned to the post-oncologic defects treatment and they became rightfully demanding in regards to the quality of reconstruction. The quest for restoring contour and form, function and beauty is not new and is not only restricted to the breast surgery. Facial defects must be reconstructed in order to regain the preoperative contouring, static and dynamic symmetry and social acceptance. Normal functions that we take for granted, like speech or swallowing must be restored for a normal life-quality when highly specialized organs like the tongue are removed. Limbs must be restored to their normal form and function for posture and ambulation.

With the advent of plastic surgery, form and function should be reestablished in the most aesthetically pleasing manner. The ideal of cosmesis expanded to the whole body. The reconstruction must take into account the expenses: donor areas should also be addressed equally. A proper functional reconstruction must not lead to a dysfunctional or conspicuous donor site.

Patients and doctors altogether became more aware of reconstruction prerequisites and oncologic patients demand no less. Plastic surgeons strive to achieve the reconstructive goals. Last, but not least, the reconstruction should be durable throughout the increasing rate of adjuvant therapies such as chemotherapy and radiation, with their sideeffects. The literature is abundant with similar examples, even though they are not termed "oncoplastic".

The case reports included herein demonstrate reconstruction of the postoncologic defects. Most patients underwent surgery associated with adjuvant therapy. The immediate or delayed reconstructive choices 
varied from skin graft to bioengineered dermal substitutes, to more complex state of the art free tissue transfer, and more elegant options such as deep tissues-sparing perforator flaps. Secondary procedures addressed the symmetry and cosmesis and the possibilities are numerous. Donor sites were not less of a concern; therefore they were addressed with equal care. Overall, oncoplastic principles were followed.
Research helps doctors help patients. To further add to the scientific level of the present Archive of Clinical Cases issue, two papers reviewed cancer research, the involvement of microorganisms in the skin tumors and the immunology related to it. Fundamental research will continue to provide a wide armamentarium to fight cancer. 